\title{
A recombinant vesicular stomatitis-based Lassa fever vaccine elicits rapid and long-term protection from lethal Lassa virus infection in guinea pigs
}

\author{
Derek R. Stein ${ }^{1}$, Bryce M. Warner ${ }^{1,2}$, Geoff Soule ${ }^{1}$, Kevin Tierney ${ }^{1}$, Kathy L. Frost ${ }^{1}$, Stephanie Booth ${ }^{1}$ and David Safronetz ${ }^{1,2}$
}

The World Health Organization has identified Lassa virus (LASV) as one of the top five pathogens to cause a severe outbreak in the near future. This study assesses the ability of a leading vaccine candidate, recombinant Vesicular stomatitis virus expressing LASV glycoprotein (VSV $\triangle$ G/LASVGPC), and its ability to induce rapid and long-term immunity to lethal guinea pig-adapted LASV (GPALASV). Outbred guinea pigs were vaccinated with a single dose of VSV $\triangle$ G/LASVGPC followed by a lethal challenge of GPA-LASV at 7, $14,25,189$, and 355 days post-vaccination. Statistically significant rapid and long-term protection was achieved at all time points with $100 \%$ protection at days 7 and 14 post-vaccination. While 83 and $87 \%$ protection were achieved at 25 days and 6 months postvaccination, respectively. When guinea pigs were challenged one year after vaccination $71 \%$ protection was achieved. Notable infectious virus was isolated from the serum and tissues of some but not all animals. Total LASVGPC-specific lgG titers were also measured on a monthly basis leading up to LASV challenge however, it is unclear if antibody alone correlates with short and long term survival. These studies confirm that a single dose of VSV $\triangle$ G/LASVGPC can induce rapid and long-term protection from LASV infection in an aggressive outbred model of infection, and supports further development in non-human primates.

npj Vaccines (2019)4:8; https://doi.org/10.1038/s41541-019-0104-x

\section{INTRODUCTION}

In December 2015, the WHO convened an expert panel to prioritize the top five emerging pathogens that are likely to cause severe outbreaks in the near future. Of the top five pathogens, Lassa virus (LASV) was identified as a priority for accelerated research and development of vaccines for which none are currently available. The newly created Coalition for Epidemic Preparedness Innovations (CEPI) has also announced significant funding for LASV vaccine research. LASV is a rodent-borne Mammarenavirus which utilizes the multi-mammate rat, Mastomys natalensis, as its natural reservoir. ${ }^{1}$ Humans are frequently exposed to LASV following direct contact with virus laden-excreta from infected rodents or through close contact with infected humans/ specimens. In humans, LASV infection can present with a wide spectrum of manifestations ranging from relatively asymptomatic or non-descript indictors of disease to severe hemorrhagic fever with multi-organ failure, a condition referred to as Lassa fever (LF). ${ }^{2}$ Estimates vary, though it is generally thought that between 300,000 and 500,000 people are infected with LASV on an annual basis, primarily in Sierra Leona, Liberia, Guinea and Nigeria making LASV one of the most prominent etiological agents of hemorrhagic fever worldwide. ${ }^{2-5}$ Although the fatality rate is considered to be between 1 and $2 \%$ of all infections, it increases dramatically in nosocomial settings and outbreaks where mortality rates exceeding $50 \%$ have been documented. ${ }^{2,5,6}$

The Vesicular stomatitis virus (VSV) recombinant vaccine platform is a leading candidate for prevention of hemorrhagic fevers in West Africa. The successful results of the VSV-Ebola (VSV $\Delta \mathrm{G}$ /
EBOVGPC) ring vaccine trials during the 2014/2015 Ebola virus outbreak underscore the potential of the vaccine as a preventative measure for hemorrhagic fevers. ${ }^{7}$ This and other ongoing safety trials point to a safe and immunogenic vaccine platform with over 20,000 participants receiving the VSV $\triangle G / E B O V G P C$ vaccine to date. Using the same vaccine platform, a VSV-based LASV vaccine (VSV $\triangle$ G/LASVGPC) was developed in parallel. ${ }^{8}$ The current VSV $\Delta G /$ LASVGPC vaccine candidate was originally designed with the glycoprotein (GPC) isolated from a Clade IV LASV (Josiah). LASV contains significant genetic heterogeneity with as many as seven clades spread across much of West Africa, making the development of a universal vaccine difficult. ${ }^{9}$ Additionally, very few studies have measured the long-term immunity and efficacy of the VSV platform, while none have specifically addressed the VSV $\Delta G$ / LASVGPC vaccine. ${ }^{10}$ While trials to examine the safety, immunogenicity, and efficacy of the VSVDG/LASVGPC vaccine would be needed, the recent EBOV vaccine trials suggest that the VSV platform will be safe and widely accepted for the prevention of hemorrhagic fever in West Africa.

Nigeria has suffered an unprecedented resurgence of LASV from 2015 to 2018. As early as April 2018, 21 states had at least one confirmed case of rodent-borne transmission. With new infections on the decline the critical phase of the outbreak is now under control. Suspected cases reached 2623 this year alone with 514 confirmed positive and 134 deaths leading to a case fatality rate of $26.1 \%$. Additionally, 39 health care workers have been infected with eight deaths. ${ }^{11}$ Coupled with high disease burden in West Africa, the potential for person-person transmission, and

\footnotetext{
'Zoonotic Diseases and Special Pathogens, National Microbiology Laboratory, Public Health Agency of Canada, Winnipeg, MB, Canada and ${ }^{2}$ Department of Medical Microbiology, University of Manitoba, Winnipeg, MB, Canada

Correspondence: David Safronetz (David.Safronetz@canada.ca)
}

Received: 14 October 2018 Accepted: 17 January 2019

Published online: 08 February 2019 

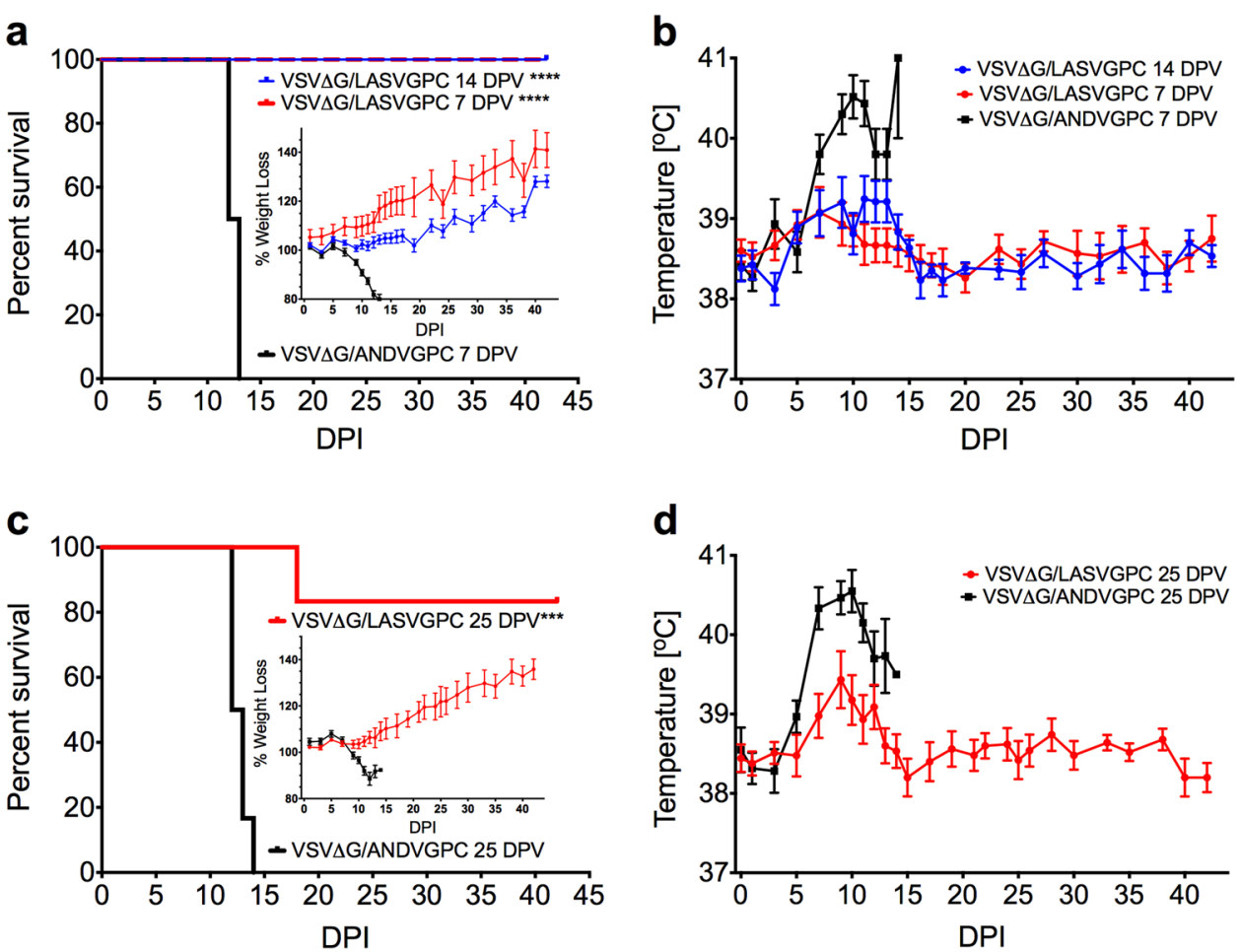

Fig. 1 Rapid protection from lethal Lassa virus challenge with a single dose of VSV $\Delta$ G-LASVGPC vaccine. a Survival, weight loss (inset) and $\mathbf{b}$ temperature analysis of Hartley guinea pigs challenged 14 and 7 days post-vaccination $(n=6)$. c Survival, weight loss (inset) and d temperature analysis of Harley guinea pigs challenged 25 days post-vaccination $(n=6)$. Mantel-Cox survival analysis was used and all data are presented as mean values with error bars indicating SEM. $p=0.1234(\mathrm{~ns}), 0.0332\left({ }^{*}\right), 0.0021\left(^{* *}\right), 0.0002\left({ }^{* * *}\right),<0.0001\left(^{* * * *}\right)$

imported cases, makes LF a significant health priority for research and development.

Historically there have been two animal models described for studying LF; inbred (strain 13) guinea pigs (GPs), and non-human primates (NHPs). ${ }^{12}$ More recently, a guinea pig-adapted (GPA) LASV model of infection has been characterized for testing vaccines and therapeutics. ${ }^{13,14}$ In this study, outbred Hartley guinea pigs were vaccinated with a single dose of VSV $\Delta \mathrm{G}$ / LASVGPC and challenged as early as 7 days or as late as 1 year after vaccination with a lethal dose of GPA-LASV; Josiah. Rapid and long-term protection from LASV challenge was achieved with sustained immunogenicity over a 1-year time span. These data indicate that the VSVIG/LASVGPC vaccine could provide rapid and sustained long-term prevention against LASV disease and mortality in West Africa.

\section{RESULTS}

Rapid protection from lethal LASV challenge with a single dose of VSV $\triangle G$-LASVGPC vaccine

In order to evaluate the ability of VSVDG-LASVGPC to rapidly protect outbred Hartley guinea pigs from lethal GPA-LASV, we vaccinated groups of nine female guinea pigs (4-5 weeks old) with $1 \times 10^{6}$ PFU of VSV $\triangle$ G-LASVGPC by the intraperitoneal route. An additional 6 control animals were vaccinated with an analogous vaccine containing Andes virus GPC (VSV $\triangle$ G-ANDVGPC) as vector control. On 7 and 14 days post-vaccination (DPV) all guinea pigs were challenged with a lethal dose of GPA-LASV $(1 \times$ $10^{4} \mathrm{TCID}_{50} / 10 \mathrm{x} \mathrm{LD}_{50}$, i.p) and monitored for signs of disease for up to 42 days (Fig. 1 a). All animals receiving the VSVDG-LASVGPC vaccine survived infection with no apparent signs of disease, and steadily gained weight throughout the experiment. The 7 and 14 DPV animals achieved $100 \%$ protection ( $p<0.0002$, Mantel-Cox) compared to control animals (VSVAG-ANDVGPC). Both VSV $\Delta$ G-
LASVGPC groups had slightly elevated temperatures as monitored by temperature transponder with an average of $39.2^{\circ} \mathrm{C}$ from days 10-14 before dropping to regular baseline measurement for the remainder of the experiment (Fig. 1b). Control animals exhibited signs of disease characterized by significant weight loss beginning on day 8 , reaching $>20 \%$ by day 14 (Fig. 1 a, inset), which was accompanied by high fever (average 40.5; 10 days post-infection), before advancing to respiratory distress and hypothermia, at which time the humane endpoints were achieved resulting in animal euthanasia. A group of three random VSVDG-LASVGPC vaccinated animals were euthanized on day 12 post-infection when control animals began to reach the humane endpoint in order to collect tissues and blood for comparison. In addition, serum was collected from all animals 10 days post-infection in order to assess viremia (Fig. 2a). The 7 and 14 DPV VSV $\Delta$ GLASVGPC animals had significantly less infectious virus in the serum compared to vector control animals on days 10 and 12 post-infection. These animals also had significantly less infectious virus in the lung (mean difference of 5 and 6.7 Log TCID ${ }_{50} / \mathrm{ml}-\mathrm{mg}$, respectively) and spleen (mean difference of 4.5 and 4.5 Log $\mathrm{TCID}_{50} / \mathrm{ml}-\mathrm{mg}$, respectively) as compared to control animals (Fig. 2a). While the samples collected from the liver had less infectious virus compared to controls it did not reach statistical significance (Supplementary Table 1). Despite the VSVDG-LASVGPC vaccine providing $100 \%$ protection from lethal disease, infectious virus was detectable in the serum, liver, lung and spleen of some animals (7 and 14 DPV) indicating that sterilizing immunity was not uniformly achieved. Total LASVGPC-specific lgG titers were assessed by endpoint dilution and the log dilution at which $90 \%$ of the sample bound LASVGPC was calculated. We found no significant difference between serum titers 5 days postvaccination between VSV $\triangle$ G-LASVGPC animals and VSV $\Delta G-$ ANDVGPC controls (Fig. 2c). However despite extremely low LASVGPC-specific IgG titers we were able to detect a significant 


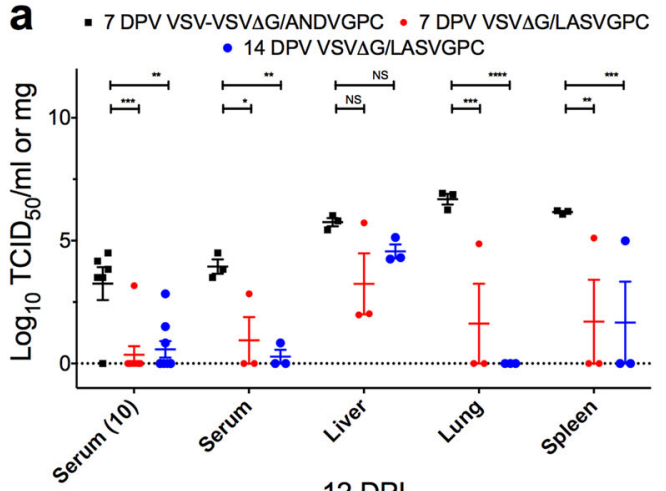

$12 \mathrm{DPI}$

C

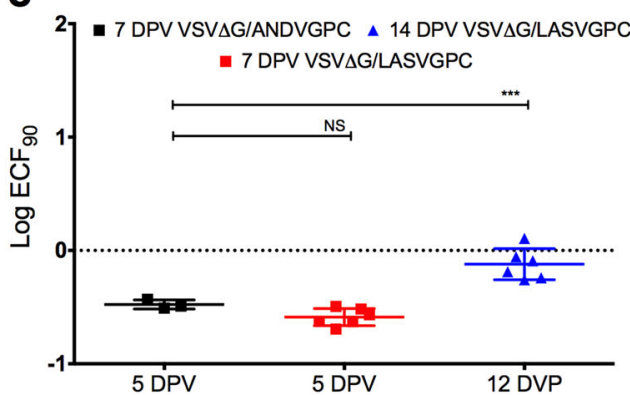

b - 25 DPV VSV $\Delta$ G/ANDVGPC • 25 DPV VSVIG/LASVGPC

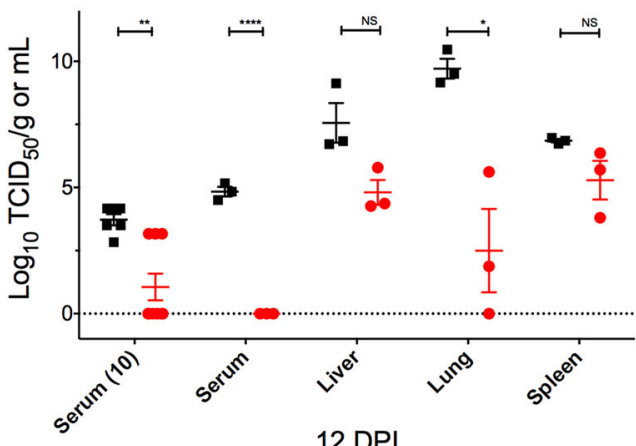

$12 \mathrm{DPI}$

d

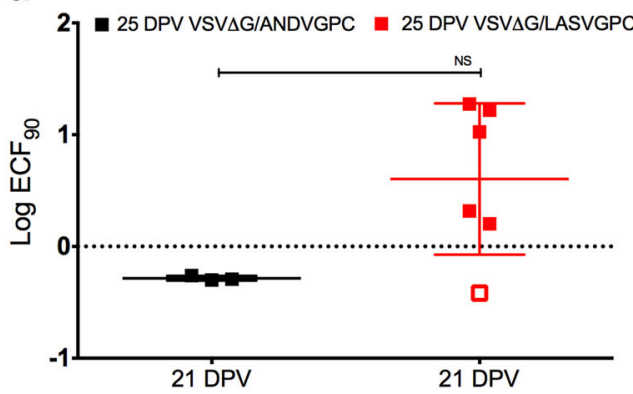

Fig. 2 Viral burden and serological analysis of rapid vaccination with VSV $\triangle G-L A S V G P C$. TCID ${ }_{50}$ analysis of serum, liver, lung and spleen collected from animals vaccinated a 7/14 DPV (2-Way Anova; Dunnett's) and b 25 DPV (T-test; Holm-Sidak). Total LASVGPC-specific lgG titers were assessed by endpoint titration and the Log $E_{C F}$ for each time-point calculated for animals challenged c 7/14 DPV (One-Way Anova; Dunnett's) and $\mathbf{d} 25$ DPV (Two-tailed T-test), respectively. Data are presented as mean values with error bars indicating SEM. $p=0.1234$ (ns), $0.03322^{(*)}, 0.00211^{(* *)}, 0.0002(* * *),<0.00011^{(* * *)}$

difference in titers 12 days post-vaccination $(p<0.0001$, One-way Anova; Dunnett's).

A second set of animals was again vaccinated with VSVDGLASVGPC and then challenged 25 DPV with a lethal dose of GPALASV in order to test the durability of protection after approximately one month. Control animals began to rapidly lose weight on day 9 post-infection (Fig. 1c, inset) which was also preceded by a rapid increase in average basal temperature beginning on day 5 post-infection. The control animals sustained a high fever from day 7 onwards reaching a peak of $40.5^{\circ} \mathrm{C}$. A rapid drop in temperature (hypothermia) coinciding with significant weight loss $(>20 \%)$ and respiratory distress resulted in humane euthanasia. In contrast, VSVDG-LASVGPC vaccinated animals steadily gained weight throughout the experiment, and generally showed no physical signs of disease with the exception of a lowgrade fever reaching a mean peak of $39.4^{\circ} \mathrm{C}$ on day 9 postinfection (Fig. 1d). The 25 DPV guinea pigs achieved statistically significant protection of $83.3 \%$ with one VSVDG-LASVGPC animal succumbing to disease (Fig. 1c, $p=0.0005$, Mantel-Cox). This single animal had delayed disease on-set compared to controls with a high-grade fever reaching a peak of $40.6^{\circ} \mathrm{C}$ by day 12 postinfection and mild weight loss (8\%). At 18 days post-infection the animal became hypothermic and showed signs of respiratory distress requiring euthanasia. Interestingly, this animal had no LASVGP-specific IgG antibody on day 21 similar to VSVAGANDVGPC controls (Fig. 2d), which could suggest a failed immunization. A group of three VSVDG-LASVGPC vaccinated animals were euthanized; serum and tissue were collected for comparison with control animals on day 12 post-infection in addition to all animals being sampled on day 10 post-infection (Fig. 2b). Control animals had infectious virus titers that were 4.8 Log $\mathrm{TCID}_{50} / \mathrm{ml}$ higher in the serum compared to VSV $\triangle \mathrm{G}$-LASVGPC vaccinated animals on day $10(p<0.0001$, Two-tailed T-test; Holm-
Sidak). As before, some VSV $\triangle$ G-LASVGPC animals had detectable viremia on day 10 (Fig. 2b). Control animals also had significantly higher titers of virus in the lung compared to VSV $\triangle$ G-LASVGPC animals with a mean difference of $7.2 \mathrm{Log} \mathrm{TCID}_{50} / \mathrm{mg}(p=0.0389$, Two-tailed T-test; Holm-Sidak). Twenty-one days post-vaccination VSVIG-LASVGPC vaccinated animals reached an average peak antibody titer of 0.47 Log $\mathrm{ECF}_{90}$ that was not significantly different than the VSV $\triangle$ G-ANDVGPC controls due to the large range/ variance in values (Fig. $2 \mathrm{~d}$ ).

Long-term protection from lethal LASV challenge with a single dose of VSV $\triangle$ G-LASVGPC vaccine

Long-term immunogenicity and protection studies with respect to the VSV vaccine platform in animal models are rare, ${ }^{10,15,16}$ owing to the extensive husbandry requirements, with none specifically studying the longevity of the VSV $\triangle$ G-LASVGPC vaccine. With this in mind we vaccinated groups of 11 guinea pigs with $1 \times 10^{6} \mathrm{PFU}$ of VSV $\triangle$ G-LASVGPC as well as an accompanying vector control vaccine (VSV $\triangle G-A N D V G P C)$. Guinea pigs were challenged 6 months (189 DPV) after vaccination with a lethal dose of GPALASV $\left(1 \times 10^{4} \mathrm{TCID}_{50}\right.$, i.p) and monitored for signs of disease for up to 42 days (Fig. 3a,b). Of the eight animals vaccinated with VSV $\Delta \mathrm{G}$ LASVGPC and challenged 6 months later, seven guinea pigs survived, leading to a statistically significant protection of $87.5 \%$ ( $p=0.0014$, Mantel-Cox). Guinea pigs that were challenged 6 months after vaccination with VSVDG-LASVGPC lost on average $10 \%$ of their starting weight by day 10 post-infection and then recovered by the end of the experiment (Fig. 3a, inset). Additionally, VSVIG-LASVGPC vaccinated animals maintained a low-grade fever from day 5-13 post-infection with a mean peak temperature of $39.3^{\circ} \mathrm{C}$ on day 11 (Fig. 3b). VSVAG-ANDVGPC control animals initially followed the same disease course, 
a

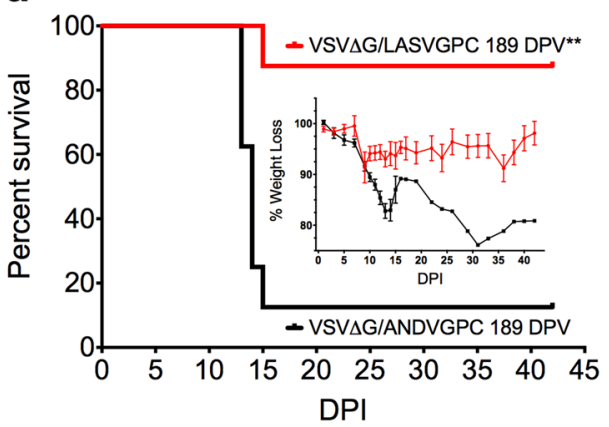

C

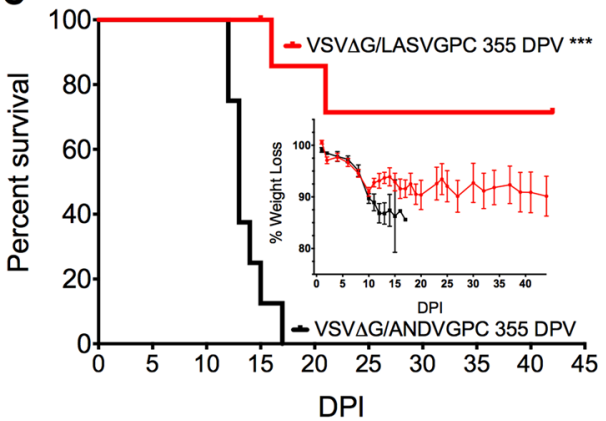

b

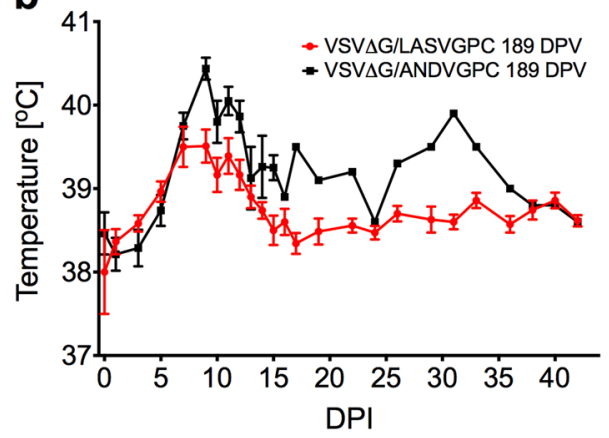

d

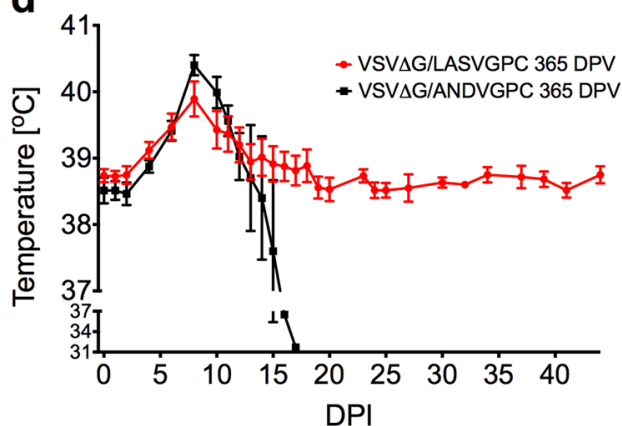

Fig. 3 Long-term protection from lethal Lassa virus challenge with a single dose of VSV $\Delta$ G-LASVGPC vaccine. a Survival, weight loss (inset) and $\mathbf{b}$ temperature analysis of Hartley guinea pigs challenged 189 days post-vaccination $(n=8)$. c Survival, weight loss (inset) and $\mathbf{d}$ temperature analysis of Harley guinea pigs challenged 355 days post-vaccination $(n=8)$. Mantel-Cox survival analysis was used and data are presented as mean values with error bars indicating SEM. $p=0.1234(\mathrm{~ns}), 0.0332\left({ }^{*}\right), 0.0021\left({ }^{* *}\right), 0.0002\left({ }^{* * *}\right),<0.0001\left({ }^{* * * *}\right)$

however they continued to lose weight 10 days post-infection, which was accompanied by a peak fever of $40^{\circ} \mathrm{C}$ on day 11 . These animals also developed hypothermia and respiratory distress requiring humane euthanasia. A single animal vaccinated with the VSV $\triangle$ G-ANDVGPC control vaccine survived lethal challenge despite seroconverting with a high IgG LASV-NP titer (Supplementary Fig. 1). In order to assess tissue related virus burden, three VSV $\triangle$ G-LASVGPC vaccinated animals were euthanized on day 13 post-infection at a time when the VSV $\triangle$ G-ANDVGPC controls were succumbing to disease. In all cases VSVDG-LASVGPC vaccinated animals had less infectious virus in the serum, liver, lung and spleen compared to controls (Fig. 4a). In particular, VSV $\Delta$ GLASVGPC vaccinated animals had a mean difference of 4.7 and 5.3 Log $\mathrm{TCID}_{50} / \mathrm{ml}-\mathrm{mg}$ isolated from the serum and liver on day 12 post-infection respectively, compared to VSV $\triangle$ G-ANDVGPC control animals.

With the need to test the long-term immunity generated by the VSV $\triangle$ G-LASVGPC vaccine we housed guinea pigs for up to one year (355 days) after a single vaccination. Animals were challenged with a lethal dose of GPA-LASV and monitored for signs of disease (Fig. 3c, d). Two out of nine VSVDG-LASVGPC vaccinated animals succumbed to infection with delayed disease onset, 16 and 21 days post-infection, respectively. A single dose of VSV $\Delta \mathrm{G}$ LASVGPC protected $71 \%$ of guinea pigs 1 year after vaccination from lethal LASV challenge ( $p=0.0001$, Mantel-Cox). On average the VSV $\triangle$ G-LASVGPC vaccinated animals lost $10 \%$ of their starting weight by day 12 , similar to those in the 6-month challenge group. Additionally they reached an average peak temperature of $39.9^{\circ} \mathrm{C}$ while the VSV $\triangle$ G-ANDVGPC controls reached an average peak temperature of $40.4{ }^{\circ} \mathrm{C}$ by day 8 post-infection. As before with the previous experiment some but not all VSVDG-LASVGPC vaccinated animals had detectable infectious virus in the serum on day 10 post-infection with a mean difference of $2.6 \mathrm{Log} \mathrm{TCID}_{50} / \mathrm{ml}$ compared to VSV $\triangle$ G-ANDVGPC controls ( $p=0.001$, Two-tailed $T$ - test; Holm-Sidak) (Fig. 4b). Tissues collected from 3 guinea pigs on day 13 post-infection also had less virus compared to controls in the liver, lung and spleen with a mean difference of $2.3,6.7$, and 4.8 Log $\mathrm{TCID}_{50} / \mathrm{mg}$, respectively (Fig. $4 \mathrm{~b}$ ).

Total IgG LASVGPC-specific antibody titers were assessed by end point dilution every month for 6 months and 1 year following vaccination (Fig. 4c, d). Almost all VSVDG-LASVGPC vaccinated animals reached peak IgG titers by day 51 which were sustained up until the final sampling date (171 or 351 DPV). However, the few animals that did succumb to disease had delayed antibody responses compared to the majority of VSVDG-LASVGPC vaccinated animals on day 21 DPV while survivors generally had higher VSV-LASVGPC IgG responses (Fig. 4c, d; dashed line).

\section{DISCUSSION}

There are numerous vaccine platforms that have shown protection in animal models against LASV infection, with one of the leading candidates being identified as the VSVDG-LASVGPC vaccine. $^{2-5,14,17-23}$ The genetic heterogeneity associated with LASV poses a significant challenge to the development of a universal vaccine to protect across a wide geographical region and multiple genotypes. However, testing of the VSVDG-LASVGPC vaccine, which was originally designed to protect against LASVJosiah (a Sierra Leone isolate) has now been shown to protect against several genetically and geographically distinct isolates. ${ }^{2,5,6,24}$ With this in mind as well as the lack of short and long-term protection studies in the literature for the VSV $\Delta$ GLASVGPC (Josiah) vaccine, we tested the ability of the vaccine to protect outbred Hartley guinea pigs from lethal LASV challenge between one week and one year following a single vaccination.

Guinea pigs challenged 7 and 14 days after VSVDG-LASVGPC vaccination were afforded $100 \%$ protection from lethal LASV infection. While animals challenged 25 days after vaccination 

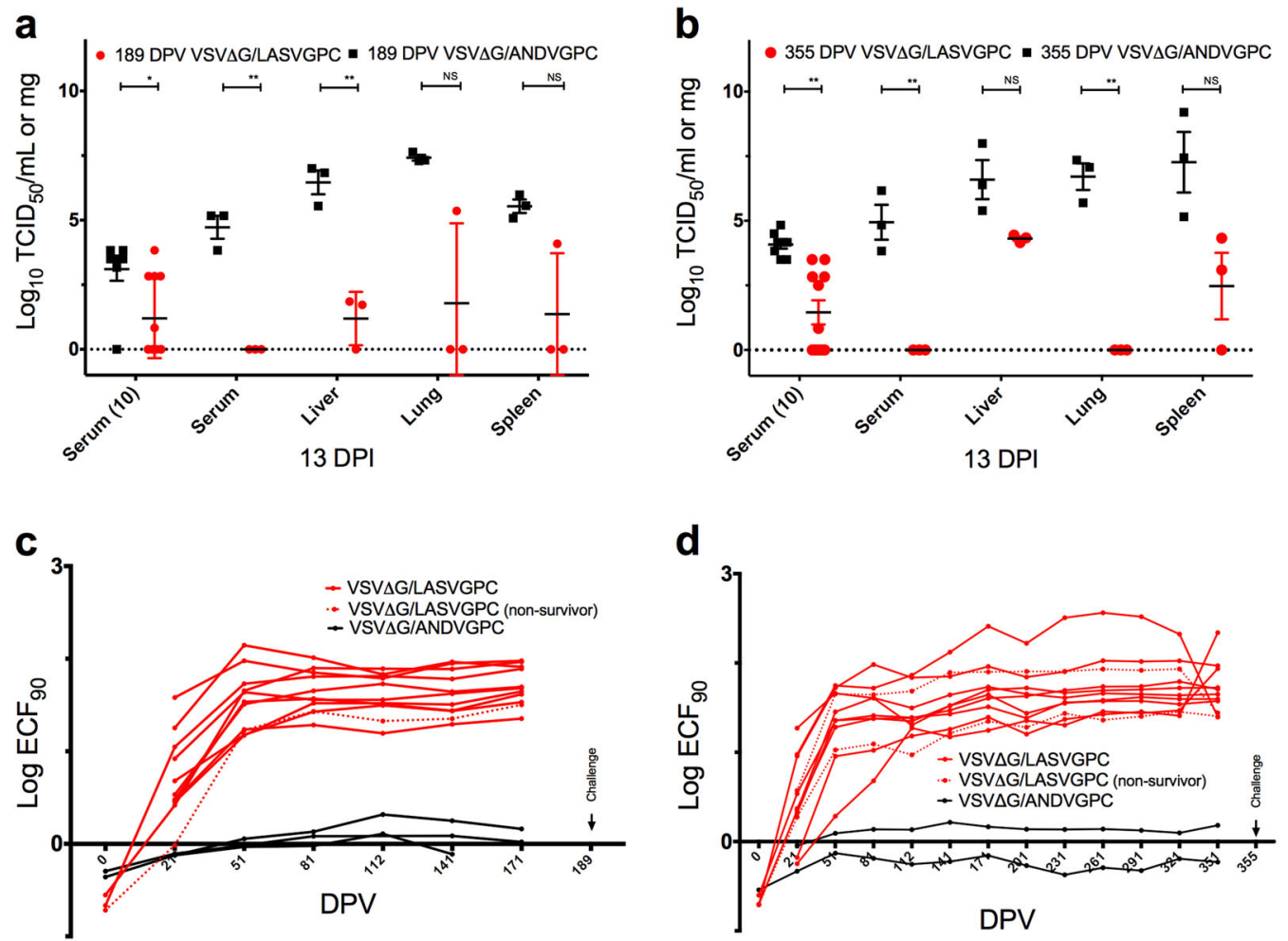

Fig. 4 Viral burden and serological analysis of long-term vaccination with VSV $\triangle$ G-LASVGPC. TCID 50 analysis of serum, liver, lung and spleen collected from animals vaccinated a 189 DPV and b 355 DPV (T-test; Holm-Sidak). Total LASVGPC-specific lgG titers were assessed by endpoint titration and the Log $\mathrm{ECF}_{90}$ for each time-point calculated for animals challenged c $189 \mathrm{DPV}$ and $\mathbf{d} 355 \mathrm{DPV}$, respectively. Data are presented as mean values with error bars indicating SEM. $p=0.1234(\mathrm{~ns}), 0.0332\left(^{*}\right), 0.0021\left(^{* *}\right), 0.0002(* * *),<0.0001\left(^{* * * *}\right)$

indicated $83.3 \%$ protection. Often, as has been shown in strain 13 guinea pigs, VSV $\triangle$ G-LASVGPC vaccination results in sterilizing immunity with no signs of clinical disease. ${ }^{7,24}$ While the protective efficacy of the vaccine remains relatively unchanged between the Strain 13 and Hartley guinea pig models, some VSVDG-LASVGPC vaccinated Hartley guinea pigs did show signs of disease including weight loss, low-grade fever, and detectable viremia 10 days postinfection. The VSV $\triangle G$-LASVGPC vaccine protective efficacy, viremia, and serology align more closely with protective data generated from NHP studies (a model that more accurately reproduces human pathogenicity). ${ }^{8,25}$ These short-term studies suggest that the GPA-LASV virus generated for testing in the outbred guinea pig model may be a more robust model of protection as compared to strain 13 guinea pigs for vaccine and therapeutic testing. A dose of $1 \times 10^{6}$ PFU was used for vaccination of all animals in our study while human and NHP studies have often used much higher doses $\left(\geq 1 \times 10^{7} \mathrm{PFU}\right)$. This discrepancy in vaccination dose may lead to sterilizing immunity or higher levels of long-term protection if doses of $\geq 1 \times 10^{7}$ PFU are used in the future. It is worth noting that all the control animals receiving VSV $\triangle G$-ANDVGPC 7 days before lethal challenge succumbed to infection, indicating a LASV-specific short term response, ruling out innate stimulation from the vector itself as seen with previous VSV-vaccine studies. ${ }^{9,26}$

When testing the long-term immunogenicity and protection of a single VSV $\triangle$ G-LASVGPC vaccination, 87.5 and $71 \%$ protection at 6 months (189 days) and 1 year (355 days) was achieved after a lethal LASV challenge, respectively. VSVDG-LASVGPC vaccinated animals that succumbed to infection had delayed time to peak antibody titers. (Fig. 4c, d; dashed lines). Interestingly on day 21 post-vaccination these animals had similar LASVGPC-specific antibody titers to the VSV $\triangle$ G-ANDVGPC vaccinated animals before eventually reaching peak titers by 51 days post-vaccination. The two non-surviving animals that were challenged 1 year after infection (Fig. 4d; dashed lines) had the lowest peak LASVGPCspecific lgG titers at the time of challenge in the VSVDG-LASVGPC vaccinated group. Analysis of LASVGPC-specific lgG in survivors and non-survivors just prior to challenge, 189 and 355 postvaccination, revealed a significant correlation between total LASVGPC-specific IgG and survival (Supplementary Fig. 2; $p=$ 0.0334 , Two-tailed $T$-test). This is in line with a recent publication identifying non-neutralizing LASVGPC-specific antibodies elicited by a LASV-Rabies vaccine as a possible correlate of protection. ${ }^{27}$ However, given that there were only 3 non-survivors for comparison in our study a larger study is warranted to evaluate if antibody titers generated by VSVDG-LASVGPC at the time of challenge correlate with survival. Recently NHP studies have shown that human neutralizing antibodies administered as late as 8 days post-infection can protect from lethal LASV infection. ${ }^{10,28}$ With this in mind we cannot rule out a role for cell-mediated protection during infection. Development of vaccines that include T-cell epitopes identified in NP or L would also be of considerable interest. Unfortunately, reagents to measure cell-mediated immune responses in the guinea pig model are lacking and limit our ability to fully assess the immunological correlates of protection in this model.

Our data suggests that the outbred Hartley guinea pig model for LASV infection could be a robust model for vaccine and therapeutic testing. While the guinea pig model for LASV infection has slightly different pathogenesis characteristics than human LF disease, protective efficacy and viremia parameters correlate closely with previous VSVDG-LASVGPC testing in NHP models of disease. ${ }^{12,25}$ Efforts should be made to adapt other geographically and genetically distinct isolates for testing of vaccine candidates in the Hartley guinea pig model. We have also shown for the first time that the VSVIG-LASVGPC vaccine can provide rapid and durable long-term protection from lethal LASV infection. Further, studies are warranted in NHPs to discover the long-term potential 
of the VSVAG-LASVGPC vaccine as a universal candidate for LF prevention in West Africa.

\section{METHODS}

Ethics

The Public Health Agency of Canada Animal Care Committee approved all animal work for this study. Certified personnel, adhering to the guidelines set forth by the Canadian Council on Animal Care (CCAC), conducted all animal procedures. All animals reaching a predetermined endpoint score were humanely euthanized in accordance with CCAC guidelines.

\section{Viruses}

Lethal infection of Hartley guinea pigs was achieved by passaging LASV Josiah in Hartley guinea pigs four times (GPA-LASV) until a uniformly lethal phenotype was achieved. ${ }^{13,14}$ The virus was propagated in VeroE6 (ATCC, CRL-1586) cells and titered using the Spearman-Karber standard $50 \%$ tissue culture infectious dose $\left(\mathrm{TCID}_{50}\right)$ method.

\section{Guinea pigs}

Four-week-old outbred female Hartley guinea pigs (Charles River, Canada) were vaccinated and housed for up to a year in groups of 6-9 animals in Biosafety Level 2 (BSL2) animal facilities before being transferred to the BSL4 environment. A minimum of one-week acclimation was observed before the start of any experimental procedures. Animals were implanted subcutaneously just prior to transfer to BSL4 with an electronic transponder temperature chip (Bio Medic Data Systems Inc.) in order to identify and monitor the animals throughout the experiment. Guinea pigs were housed in caging units that provide negative pressure and HEPAfiltered containment in the National Microbiology Laboratory BSL4 for LASV challenge.

\section{In-vivo efficacy studies}

All guinea pigs were vaccinated with either a single dose of recombinant VSV $\triangle$ G-ANDVGPC as a control or VSV $\triangle$ G-LASVGPC $\left(1 \times 10^{6}\right.$ PFU) by the intraperitoneal route. Guinea pigs were housed for 7 (9/group), 14 (9/ group), 25 (9/group), 189 (11/group), and 355 (11/group) days postvaccination. Guinea pigs were sampled 1 week, 3 weeks, and every month thereafter following vaccination in order to assess total LASVGP-specific IgG titers. On the respective time points, animals were transferred to the BSL4 facility and challenged with a lethal dose of guinea pig-adapted LASV $\left(1 \times 10^{4} \quad \mathrm{TCID}_{50} / 10 \times \mathrm{LD}_{50}\right)$ by the intraperitoneal route. Animals were weighed and temperatures were taken on a daily basis in addition to daily clinical scoring. At a time when control (VSV $\triangle$ G-ANDVGPC immunized GPs) animals experienced advanced signs of disease requiring euthanasia, 3 random guinea pigs from the VSVAG-LASVGPC vaccinated groups were euthanized to compare viral titers, and tissue specific replication. The remaining guinea pigs were monitored for survival until day 42 postinfection or until endpoint scoring was achieved requiring euthanasia.

\section{$\mathrm{TCID}_{50}$ determination}

VeroE6 cells were seeded into 96-well tissue culture plates in order to become $70-80 \%$ confluent within $24 \mathrm{~h}$. Tissues were homogenized in $1 \mathrm{ml}$ of DMEM using a TissueLyserll (Qiagen). The homogenates were clarified by centrifugation at $10,000 \times g$ for $10 \mathrm{~min}$. Clarified homogenates or serum were ten-fold serial diluted in DMEM containing 2\% FBS and $100 \mu \mathrm{l}$ of each dilution in triplicate were added per well. Cells were then incubated for 7 days at $37^{\circ} \mathrm{C}$ with $5 \% \mathrm{CO}_{2}$. Cells were then assessed for virus-induced cytopathic effect and the tissue culture infectious dose $\left(\mathrm{TCID}_{50}\right)$ was calculated by the Spearman-Karber method. ${ }^{29}$

\section{Lassa virus serology and seroconversion}

In order to measure the total IgG LASVGPC-specific immune responses serum was collected once a month from all vaccinated animals. Additionally to ensure surviving guinea pigs received an adequate challenge, serum samples were collected from all surviving animals at day 42 post-infection. A commercial ReLASV ${ }^{\mathrm{TM}}$ Pan-Lassa GP or NP IgG/lgM ELISA (Zalgen Labs) was modified to measure total guinea pig-specific lgG to LASV GP or NP as follows. Briefly, guinea pig serum samples were diluted 1 in 100 in order to measure NP specific responses and incubated for $30 \mathrm{~min}$ at room temperature on pre-coated ELISA plates with recombinant nucleoprotein. Plates were washed four times with the kit provided wash buffer. A secondary goat anti-guinea pig specific lgG (H\&L)HRP (SeraCare) antibody replaced the kit provided antibody and was diluted at 1:1000. Plates were again incubated for $30 \mathrm{~min}$ at room temperature followed by 4 washes. The kit provided one-component substrate was added to the plates and incubated for $10 \mathrm{~min}$. The reaction was stopped using $0.36 \mathrm{~N}$ sulfuric acid and the $\mathrm{OD}$ of samples read at $405 \mathrm{~nm}$.

\section{Statistical analysis}

Statistical analyses were conducted using GraphPad Prism 7.0. A log rank Mantel-Cox test was used for survival analysis. Infectious virus comparisons in the serum and tissues were performed with either a 2-way Anova or multiple $t$-tests corrected for multiple comparisons by the Holm-Sidak method. $p$ values of $<0.05$ were considered significant.

\section{Reporting summary}

Further information on research design is available in the Nature Research Reporting Summary linked to this article.

\section{DATA AVAILABILITY}

The authors declare that all data supporting the findings of this study are available within the paper and supplementary files.

\section{ACKNOWLEDGEMENTS}

The authors would like to acknowledge the staff of the veterinary technical services division at the National Microbiology Laboratory, in particular, Stephanie Kukas, Christine DeGraff, Kimberley Azaransky, Michelle French, and Julie Kubay. Funding was provided by the Public Health Agency of Canada.

\section{AUTHOR CONTRIBUTIONS}

D.R.S. and D.S. designed and directed the experiments contained in this manuscript. B.M.W. and G.S. performed infectious titer analysis while K.T. provided animal technician support. K.L.F. and S.B. performed data analysis. D.R.S. conducted the data analysis and manuscript preparation.

\section{ADDITIONAL INFORMATION}

Supplementary information accompanies the paper on the npj Vaccines website (https://doi.org/10.1038/s41541-019-0104-x).

Competing interests: The authors declare no competing interests.

Publisher's note: Springer Nature remains neutral with regard to jurisdictional claims in published maps and institutional affiliations.

\section{REFERENCES}

1. Monath, T. P., Newhouse, V. F., Kemp, G. E., Setzer, H. W. \& Cacciapuoti, A. Lassa virus isolation from mastomys natalensis rodents during an epidemic in Sierra Leone. Science 185, 263-265 (1974).

2. McCormick, J. B. et al. A case-control study of the clinical diagnosis and course of lassa fever. J. Infect. Dis. 155, 445-455 (1987).

3. Frame, J. D. Clinical features of lassa fever in liberia. Rev. Infect. Dis. 11, S783-S789 (1989).

4. Bausch, D. G. et al. Lassa fever in guinea: I. Epidemiology of human disease and clinical observations. Vector-Borne Zoonotic Dis. 1, 269-281 (2001).

5. Ogbu, O., Ajuluchukwu, E. \& Uneke, C. J. Lassa fever in West African sub-region: an overview. J Vector Borne 44, 1-11 (2007).

6. Fisher-Hoch, S. P., Tomori, O. \& Nasidi, A. Review of cases of nosocomial Lassa fever in Nigeria: the high price of poor medical practice. BMJ 311, 857-859 (1995).

7. Henao-Restrepo, A. M. et al. Efficacy and effectiveness of an rVSV-vectored vaccine expressing Ebola surface glycoprotein: interim results from the Guinea ring vaccination cluster-randomised trial. Lancet 386, 857-866 (2015). 
8. Garbutt, M. et al. Properties of replication-competent vesicular stomatitis virus vectors expressing glycoproteins of filoviruses and arenaviruses. J. Virol. 78, 5458-5465 (2004).

9. Warner, B. M., Safronetz, D. \& Stein, D. R. Current research for a vaccine against Lassa hemorrhagic fever virus. DDDT 12, 2519-2527 (2018).

10. Wong, G. et al. Immunization with vesicular stomatitis virus vaccine expressing the Ebola glycoprotein provides sustained long-term protection in rodents. Vaccine 32, 5722- 5729 (2014).

11. An update of Lassa fever outbreak in Nigeria. NCDC 1-4 (2018).

12. Gowen, B. B. \& Holbrook, M. R. Animal models of highly pathogenic RNA viral infections: hemorrhagic fever viruses. Antivir. Res. 78, 79-90 (2008).

13. Safronetz, D. et al. The broad-spectrum antiviral favipiravir protects guinea pigs from lethal Lassa virus infection post-disease onset. Sci. Rep. 5, 14775 (2015).

14. Cross, R. W. et al. Treatment of Lassa virus infection in outbred guinea pigs with first-in-class human monoclonal antibodies. Antivir. Res. 133, 218-222 (2016).

15. Mire, C. E. et al. Durability of a vesicular stomatitis virus-based marburg virus vaccine in nonhuman primates. PLoS One 9, e94355 (2014).

16. Fausther-Bovendo, H., Alimonti, J. B. \& Kobinger, G. P. Immunization with vesicular stomatitis virus vaccine expressing the Ebola glycoprotein provides sustained long-term protection in rodents. Vaccine 32, 5722-5729 (2014).

17. Pushko, P., Geisbert, J., Parker, M., Jahrling, P. \& Smith, J. Individual and bivalent vaccines based on alphavirus replicons protect guinea pigs against infection with Lassa and Ebola viruses. J. Virol. 75, 11677-11685 (2001).

18. Wang, M., Jokinen, J., Tretyakova, I., Pushko, P. \& Lukashevich, I. S. Alphavirus vector-based replicon particles expressing multivalent cross-protective Lassa virus glycoproteins. Vaccine 36, 683-690 (2018).

19. McCormick, J. B., Mitchell, S. W., Kiley, M. P., Ruo, S. \& Fisher-Hoch, S. P. Inactivated Lassa virus elicits a non protective immune response in rhesus monkeys. J. Med. Virol. 37, 1-7 (1992).

20. Cashman, K. A. et al. Enhanced efficacy of a codon-optimized DNA vaccine encoding the glycoprotein precursor gene of Lassa virus in a Guinea Pig Disease Model when delivered by dermal electroporation. Vaccines 1, 262-277 (2013).

21. Cashman, K. A. et al. DNA vaccines elicit durable protective immunity against individual or simultaneous infections with Lassa and Ebola viruses in guinea pigs. Hum. Vaccin Immunother. 13, 3010-3019 (2017).

22. Lukashevich, I. S., Vasiuchkov, A. D., Stel'makh, T. A., Scheslenok, E. P. \& Shabanov, A. G. The isolation and characteristics of reassortants between the Lassa and Mopeia arenaviruses. Vopr. Virusol. 36, 146-150 (1991).
23. Lukashevich, I. S. et al. Safety, immunogenicity, and efficacy of the ML29 reassortant vaccine for Lassa fever in small non-human primates. Vaccine $\mathbf{2 6}$ 5246-5254 (2008).

24. Safronetz, D. et al. A recombinant vesicular stomatitis virus-based Lassa fever vaccine protects guinea pigs and macaques against challenge with geographically and genetically distinct Lassa viruses. PLoS Negl. Trop. Dis. 9, e0003736 (2015).

25. Geisbert, T. W. et al. Development of a new vaccine for the prevention of Lassa fever. PLoS Med. 2, e183 (2005).

26. Brown, K. S., Safronetz, D., Marzi, A., Ebihara, H. \& Feldmann, H. Vesicular stomatitis virus-based vaccine protects hamsters against lethal challenge with Andes virus. J. Virol. 85, 12781-12791 (2011).

27. Abreu-Mota, T. et al. Non-neutralizing antibodies elicited by recombinant LassaRabies vaccine are critical for protection against Lassa fever. Nat. Commun. 9 4223 (2018).

28. Mire, C. E. et al. Human-monoclonal-antibody therapy protects nonhuman primates against advanced Lassa fever. Nat. Med. 23, 1146-1149 (2017).

29. Ramakrishnan, M. A. Determination of $50 \%$ endpoint titer using a simple formula. World J. Virol. 5, 85-86 (2016).

(i) Open Access This article is licensed under a Creative Commons Attribution 4.0 International License, which permits use, sharing, adaptation, distribution and reproduction in any medium or format, as long as you give appropriate credit to the original author(s) and the source, provide a link to the Creative Commons license, and indicate if changes were made. The images or other third party material in this article are included in the article's Creative Commons license, unless indicated otherwise in a credit line to the material. If material is not included in the article's Creative Commons license and your intended use is not permitted by statutory regulation or exceeds the permitted use, you will need to obtain permission directly from the copyright holder. To view a copy of this license, visit http://creativecommons. org/licenses/by/4.0/.

(C) Crown 2019 